\title{
Deformation dependencies of sand stiffness
}

\author{
Tomas Sabaliauskas ${ }^{1, *}$ and Lars Bo Ibsen ${ }^{1}$ \\ ${ }^{1}$ Aalborg University, Thomas Manns vej 23, 9220, Aalborg, Denmark
}

\begin{abstract}
Convention does not provide a solution combining drained and undrained response, loss and recovery of sand stiffness, all in one irregular loading sequence. Alas, such solution is necessary for designing offshore wind turbine foundations and predicting soil behavior during (and after) seismic events.

To gain deeper understanding of the fundamental phenomenon governing disturbed sand behavior, unconventional testing procedures were attempted using a frictionless triaxial apparatus. The resulting observations revealed a new physical phenomenon (deformation dependent stiffness hysteresis loops). The new observations were then used to derive an unconventional model (BSM), which uses a strain (deformation) envelope.
\end{abstract}

\section{Introduction}

Classical geotechnics focuses on quasi-static loading cases, such as slope stability and static structures (buildings or dams). There, dynamic / cyclic loads don't play a dominant role. More recent research analyses seismic events, where cyclic loads occur in short bursts of large energy. Offshore wind turbines raise a different challenge, as their foundations are exposed to continuous cyclic loads generated by wind, waves and moving components [1]. Cyclic loads can cause both positive and negative effects: disturb or stabilize the foundation, reduce or increase the stiffness of soil. Especially during extreme events - storms, collisions, emergency stops [1]. Therefore, it is necessary to investigate the fundamental phenomenon governing loss and recovery of disturbed sand stiffness, during both drained and undrained loading, under irregular loading cycles.

Contemporary convention lacks consensus on how to treat cyclic preloading effects [2]. Conventional tests fail to observe sand stiffness at its full complexity [3]. Procedures presented in this paper are different, as they attempted to combine both: drained and undrained response, both: loss and recovery of stiffness, during irregular loading cycles - all under one set of rules, in an original model called BSM (Bezier stiffness model). The model is radically different from convention, as it is anchored to a deformation envelope rather than stress space parameters. BSM was derived directly from novel experimental observations [4] [5], rather than building on top of existing convention.

The BSM is meant to capture the long term behavior of disturbed sand. Unlike conventional models, BSM does not depend on "initial state". Instead, BSM predicts evolution of disturbed soil states. It converges towards the "final" shape of the stiffness hysteresis loop, regardless of "initial state". In addition, the novel approach has remarkable prescriptive power, which allows to control real life

*e-mail: ts@ civil.aau.dk specimens in real life testing. The specimen stiffness can be reduced and increased selectively. The model can capture even a paradoxical soil state demonstrated experimentally: lower stiffness at higher density [6] [5]. Thus, proving deep fundamental compatibility between the numerical model, and physical properties of the real material.

BSM has the potential for a wide scope of applications. For example: designing anchors of ships or other floating structures. Similar models could predict disturbed soil states as they develop before, during and after an earthquake. BSM could allow to control soil properties with greater precision in industrial processes, where targeted volumes of sand are modified intentionally towards higher / lower stiffness. The wide scope of potential applications comes from the fact that fundamental soil properties are explored - stiffness hysteresis loops. Therefore, touching all areas of geotechnics where sand stiffness is disturbed by cyclic loads.

\section{The new modeling principle (BSM)}

The method of Bezier stiffness model (BSM) uses a defamation (strain-space) envelope. The solution starts by checking position of a strain (deformation) within the strain (deformation) envelope. Then, deviator stress $q$ is interpolated within the Coulomb stress limits (Eq's. 4, 5 ) in proportion to $\epsilon$ position within the deformation envelope. Thus, the stress point always stays within the Coulomb envelope limits. In the model, $q$ (deviator stress) is described using polar coordinates (Eq.2). To find $q$ using polar coordinates, two lines are used: a line following the stress angle $\eta=q / p$, and a line following the effective stress path (ESP) (see Fig.2). The two lines perform independently of each other, and are governed by two mutually independent variables ( $\eta$ governed by $\epsilon$ position within the deformation envelope, ESP governed by $\sigma_{r}$ value). The exact $q$ value is where the two lines cross each other. 


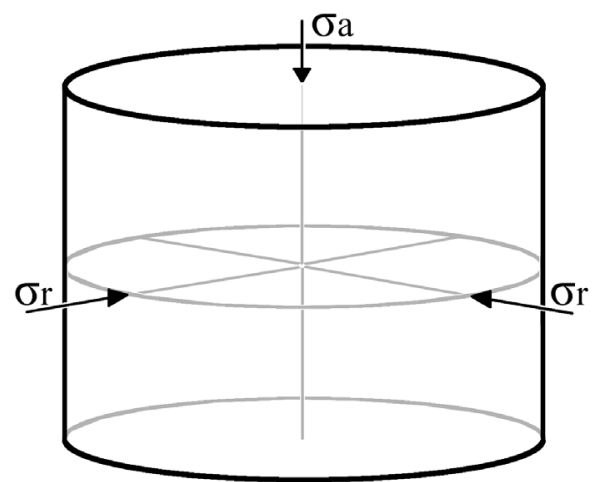

Fig. 1. Geometry of the specimen, tested using the frictionless triaxial apparatus. In this paper the BSM is used to model behavior of a sand specimen (Frederikshavn sand), which has height to diameter ratio $1: 1$, and is loaded using end plates with low friction [3] [6].

The effective radial stress $\left(\sigma_{r}\right)$, shown in Fig. 1 is constant during drained triaxial testing. Thus, $\sigma_{r}$ can be held constant when doing simulations of drained specimens using BSM. In undrained test, however, $\sigma_{r}$ varies due to pore pressure variation, as the absolute radial stress is carried by both pore water pressure and effective stress. Undrained specimen contract and dilate, causing pore water pressure to vary, thus casing effective $\sigma_{r}$ variation. Undrained pore pressure variation is an unresolved nonlinear problem in geotechnics. Therefore, during undrained triaxial test simulations, $\sigma_{r}$ values have to be borrowed from the measurement history. Nevertheless, $\eta$ behaves as a function of $\epsilon$ at all times, regardless of drained or undrained mode.

$$
\begin{array}{r}
p=\frac{\sigma_{a}+2 \cdot \sigma_{r}}{3} \\
q=\sigma_{a}-\sigma_{r} \\
\eta=q / p \\
q_{\text {max }}=-\frac{3 \cdot \sigma_{r} \cdot \tan (\phi)}{\tan (\phi)-1} \\
q_{\text {min }}=-\frac{3 \cdot \sigma_{r} \cdot \tan (\phi)}{\tan (\phi)+1}
\end{array}
$$

$\eta$ is modeled as a function of $\epsilon$, with respect to $\epsilon$ position within the deformation envelope ( $\epsilon_{y}$, Eq. 6). The $\epsilon_{y}$ is defined in strain space coordinates. For a triaxial test, the $\epsilon_{y}$ boundaries move along unidimensional $\epsilon$ axis. The $\epsilon_{y}$ envelope can be described as a circle, with the center and radius coordinate (see Fig.3). During simulation, $\epsilon_{y}$ radius and center change in proportion to strain increment $\left(\Delta \epsilon_{y}\right)$ (Eq's 7,8). The radius can increase and decrease (isotropic hardening), and the center coordinate can move as well (kinematic hardening). If the applied $\epsilon$ increment crosses $\epsilon_{y}$ boundary $\left(f_{\epsilon}<0\right)$, then yielding is caused. When yielding, $\epsilon_{y}$ expands to touch the $\epsilon$ position. Thus, keeping $\epsilon$ within $\epsilon_{y}$ limits at all times. Otherwise (when not yielding) $\epsilon_{y}$ envelope shrinks in proportion to $\Delta \epsilon$ (see Fig.3). Thus, there are no "pure elastic" steps in BSM model. The size and position of $\epsilon_{y}$ changes with every $\Delta \epsilon$, in proportion to
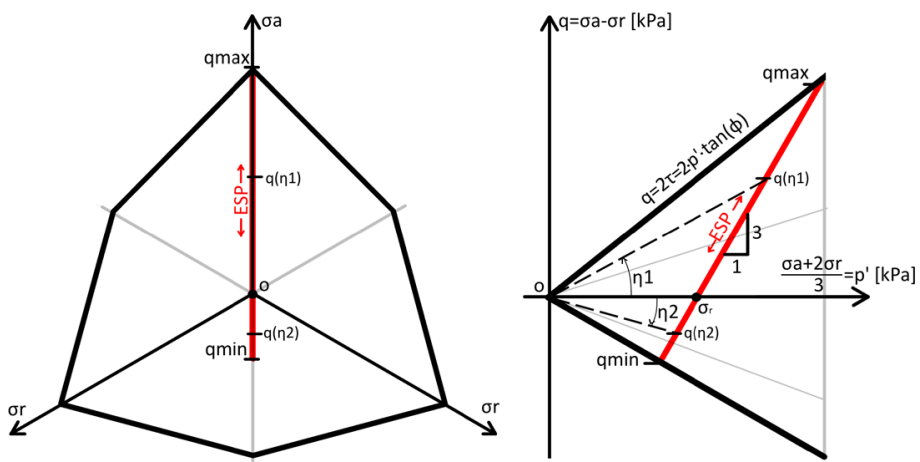

Fig. 2. The $\eta$ and $\sigma_{r}$ are independent. $\eta$ angle is controlled by interpolating $\epsilon$ within the deformation envelope. $\sigma_{r}$ is input by user, it controls the position of the ESP line. Where ESP and $\eta$ lines cross, the $q$ is found.

$\kappa$ coefficients. If $\epsilon$ cycles are applied within the $\epsilon_{y}$ limits, the $\epsilon_{y}$ shrinks gradually, until the edge of the $\epsilon_{y}$ begins to touch the peaks of applied $\epsilon$ cycles. Consequently, when cycles of constant $\epsilon$ amplitude are applied, $\epsilon_{y}$ will converge to match the peaks of applied deformation amplitude. This is a very important feature, as stiffness hysteresis loops in sand converge towards a predictable pattern, when deformation cycles of constant amplitude are applied [6] [5]. Thus, because the envelope adapts to match applied deformation amplitude, a stiffness hysteresis loop normalized within the envelope will overlap the stiffness hysteresis loop generated experimentally.

$$
f_{\epsilon}=\epsilon_{R}-a b s\left(\epsilon-\epsilon_{o}\right)
$$

$$
\begin{array}{r}
\Delta \epsilon_{R}= \begin{cases}\kappa_{R, 1} \cdot a b s(\Delta \epsilon), & \text { if } f_{\epsilon} \leq 0 \text { stabilize } \\
\kappa_{R, 2} \cdot f_{\epsilon}, & \text { if } f_{\epsilon}>0 \text { yielding }\end{cases} \\
\Delta \epsilon_{o}= \begin{cases}\kappa_{O, 1} \cdot \Delta \epsilon, & \text { if } f_{\epsilon} \leq 0 \text { stabilize } \\
\kappa_{O, 2} \cdot f_{\epsilon}, & \text { if } f_{\epsilon}>0 \text { yielding }\end{cases}
\end{array}
$$

Within the $\epsilon_{y}$ envelope hardening curves are normalized (Eq.9, Fig.4). The curves were obtained by allowing stiffness hysteresis loops to stabilize at different deformation amplitudes during real life triaxial testing [6] [5]. The curves were obtained at different deformation amplitudes and $\sigma_{r}$ values, then normalized between 1 and 0 along both axes.

Since the hardening curve is normalized within $\epsilon_{y}$ envelope, when $\epsilon_{y}$ expands (yielding), the stiffness curves become stretched (lower stiffness). The process of losing stiffness this way will be referred to as "disturbing" the sand (disturbed sand has lower stiffness). On the other hand, when $\epsilon_{y}$ envelope shrinks, the stiffness hysteresis loops become steeper. Thus the specimen recovers stiffness. The process of stiffness recovery will be referred to as "stabilizing". A real sand specimen, during a real frictionless triaxial test, can be repeatedly stabilized and disturbed towards different deformation amplitudes [4] [5], [6], and the BSM model captures the behavior by adjusting $\epsilon_{y}$ towards applied deformation history. 


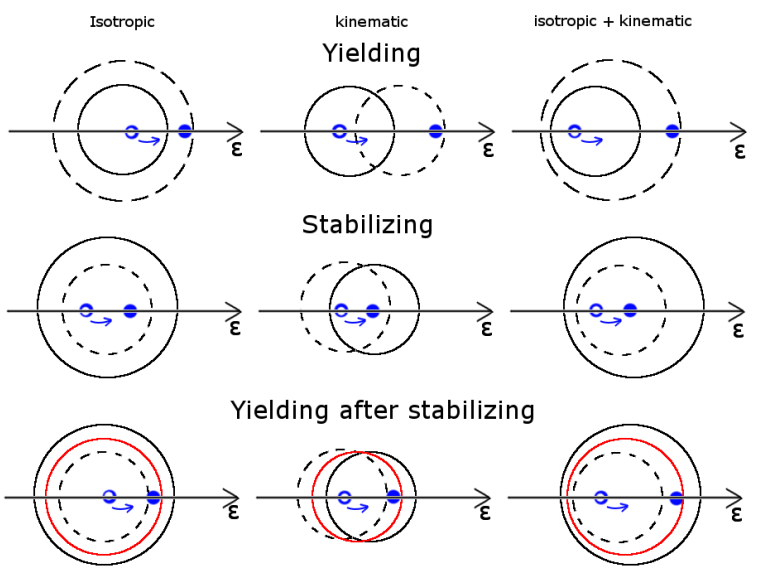

Fig. 3. Combinations of kinematic / isotropic hardening (change in radius and/or position) of the deformation envelope. When the dot moves, envelope stabilizes. If the dot is outside the envelope, the envelope yields - to contain the dot inside.

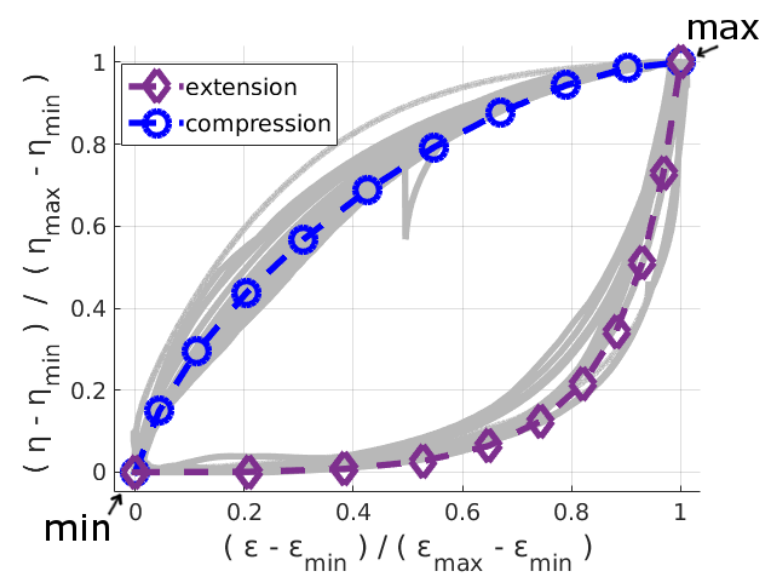

Fig. 4. Hardening curves from drained triaxial tests. Deformation cycles of various amplitude applied until stiffness hysteresis converged [6] [4] [5]. The converged hysteresis loops were normalized with respect to $\eta$ and $\epsilon$.

$\eta_{\text {norm }}(x)= \begin{cases}-\left((9-8 x)^{\frac{1}{2}}-3\right)^{\frac{3}{8}} & , \text { for }\left(\eta_{\min }\right) \\ \frac{3(40 x+9)^{\frac{1}{2}}}{8}-\left(\frac{5\left(\frac{2 x}{5}+\frac{9}{100}\right)^{\frac{1}{2}}}{2}-\frac{3}{4}\right)^{\frac{3}{2}}-\frac{9}{8} & , \text { for }\left(\eta_{\max }\right)\end{cases}$

During $\Delta \epsilon>0$ (compression), path $\eta_{\max }$ is followed, given $\Delta \epsilon<0$ (extension phase), $\eta_{\min }$ is followed. This is enough to approximate the shape and position of the hysteresis loop towards which a specimen will converge. However, convergence is gradual, and during it the unloading / reloading $\eta$ should not "teleport" between extension and compression paths. A method to gradually transition from one hardening curve to the other is necessary. For that purpose the Bezier stiffness principle is introduced (see Fig.5, and Eq's. 10-12).

Bezier stiffness borrows a principle used in vector graphics - Bezier splines (see Fig.5). $K_{b}$ has finite steepness (Eq.10), and is parametrized by $R(x)$ (Eq. 12). If the

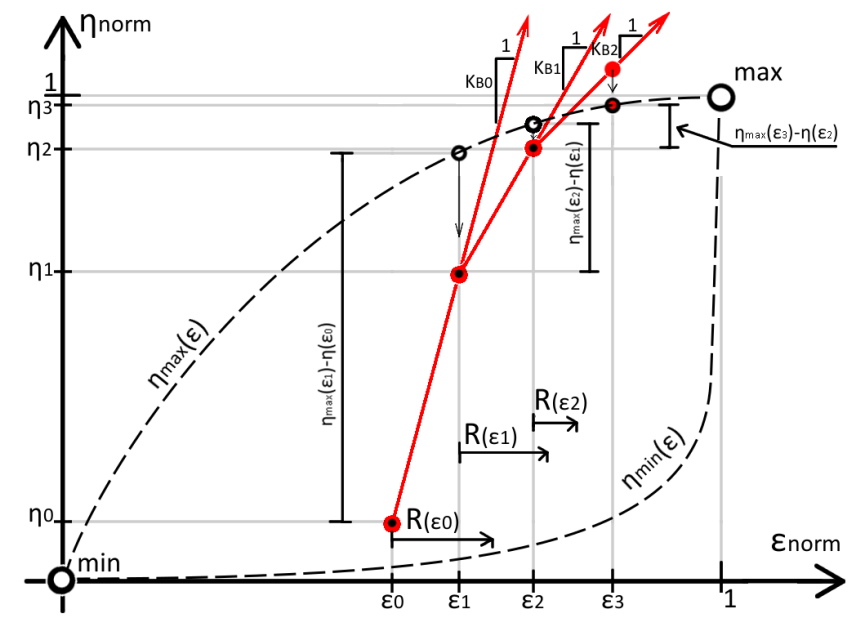

Fig. 5. Bezier stiffness principle.

Table 1. Solution algorithm

1. Inputs: $\epsilon, \sigma_{r}$.
2. Kinematic - isotropic update of $\epsilon_{y}$ (Eq's. 7,8$)$.
3. If yielding (Eq.6), update $\epsilon_{y}=$ epsilon.
4. Interpolate $\epsilon$ within $\epsilon_{y}$.
5. Bezier stiffness step, to obtain $\eta$.
6. Output $q$. (Eq. 4, using $\eta$ in place of $\phi)$.

$\Delta \epsilon$ is small (say $\epsilon_{0}$ to $\epsilon_{1}$ in Fig.5), then a step with inclination $K_{b}$ is taken, which results in $<\eta_{\min }<\eta<\eta_{\max }$. If the step size is relatively big (see step $\epsilon_{2}-\epsilon_{3}$ ), then the "predictor" $\eta$ value reaches beyond the $\eta_{\max }$. In which case a correction $\left(\eta=\eta_{\text {max }}\right)$ is made .

At this point all the components for implementing BSM were described. The deformation envelope and kinematic / isotropic rules governing the envelope. The origin and implementation of hardening curves normalized within the envelope. And a method of interpolating within the hardening curves (Bezier stiffness). Thus, given $\epsilon$ and sigma $_{r}$, the $q$ value can be found (see the algorithm in table $1)$.

$$
\begin{array}{r}
K_{b}(i)=\frac{\eta_{\max }(\epsilon(i+1))-\eta(i)}{R(\epsilon(i))} \\
\eta(i+1)=\eta(i)+K_{B}(i) \Delta \epsilon \\
R(x)=\frac{1-(1-2 x)^{2}}{10}+10^{-4}
\end{array}
$$

\section{Simulation results}

Performance of BSM is compared to sand specimen response measured using a frictionless triaxial apparatus. The loading sequences in the testing procedures are unconventional. Large deformation amplitudes are applied, specimen peak strength is tested more than once in one loading sequence. Stiffness is repeatedly reduced and increased (disturbed and recovered) numerous times in one sequence. Drained and undrained loading cycles are combined into one irregular loading sequence. And the BSM 

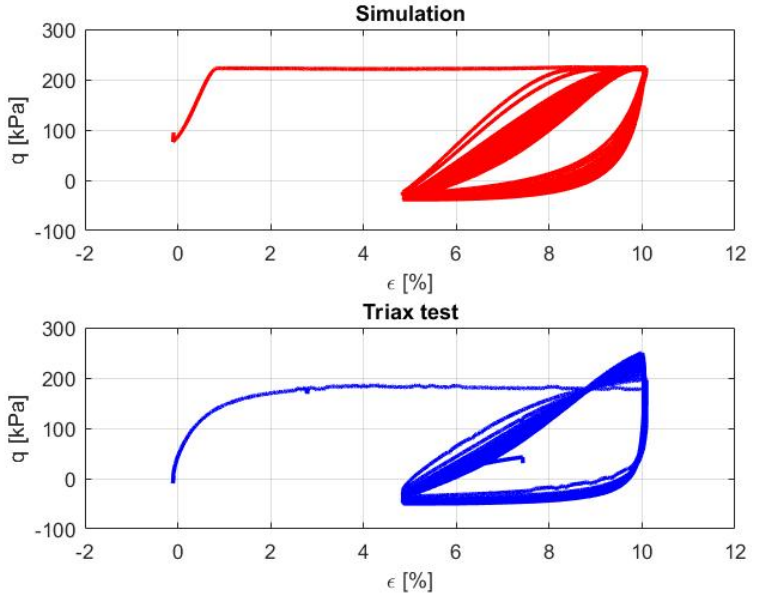

Fig. 6. Simulation and triaxial test where 5\% amplitude deformation cycle is applied.

model is shown to be representative of a large portion of observations generated experimentally.

The case specific tests compared with BSM here were executed on Frederkishavn sand. Similar tests had been done on Baskarp sand, Aalborg University sand no.1, silt and gravel [6] [4] [5]. Thus, the stiffness hysteresis loop behavior is not unique to Frederikhavn sand, and stiffness hysteresis loop patterns captured by BSM are common to a wide range of frictional soils.

The simulations shown in Fig's.6-Fig.10 are all made using BSM with the same calibration factors: $\kappa_{R, 1}=0.08$, $\kappa_{R, 2}=0.5, \kappa_{O, 1}=0.04, \kappa_{O, 2}=0.5$. The friction angle is treated as constant $\phi=40$. The initial position of $\epsilon_{y}$ envelope is $\epsilon_{0}=0, \epsilon_{R}=0.001$.

In Fig. 6 the deformation envelope is challenged to converge towards $5 \% \epsilon$ amplitude cycles. During the simulation the stiffness triangle gradually converges from concave to convex curve. Alas, in simulation the peak of the stiffness triangle is not growing. The measured increase in peak strength can be attributed to increasing density [6]. Thus, to capture it, the simulation would require a method of modeling volumetric response during irregular loading. In addition, the deformation envelope moves at all times. This is a problem, because the hardening curves were normalized while assuming a constant radius and position of the envelope. This leads to a slightly distorted stiffness hysteresis loop, the shape of which depends on the isotropic / kinematic parameters. Nevertheless, despite imperfections, the simulation does predict the correct position of the converging stiffness triangle.

In Fig.7 the deformation envelope is challenged to converge towards $1 \% \epsilon$ amplitude cycles, before "exiting" the triangle through compression and applying a 3\% $\epsilon$ amplitude. During the simulation the first stiffness triangle converges correctly, and the next loading cycles is correctly predicted to have lower stiffness. Changes in peak strength are ignored, as strength parameter $\phi$ is held constant. The increase in peak strength is likely due to changes in den-
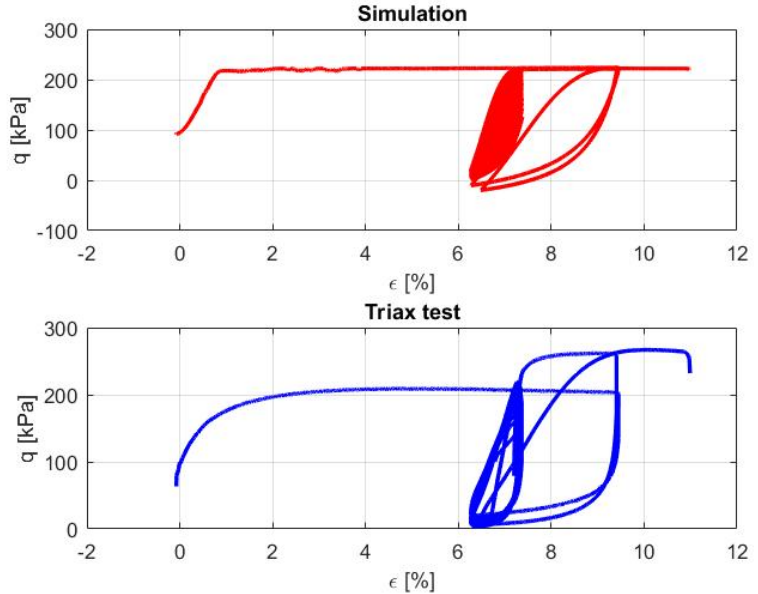

Fig. 7. Simulation and triaxial test where $1 \%$ deformation cycles (stabilizing the stiffness triangle) are followed by one cycle of $3 \%$ amplitude
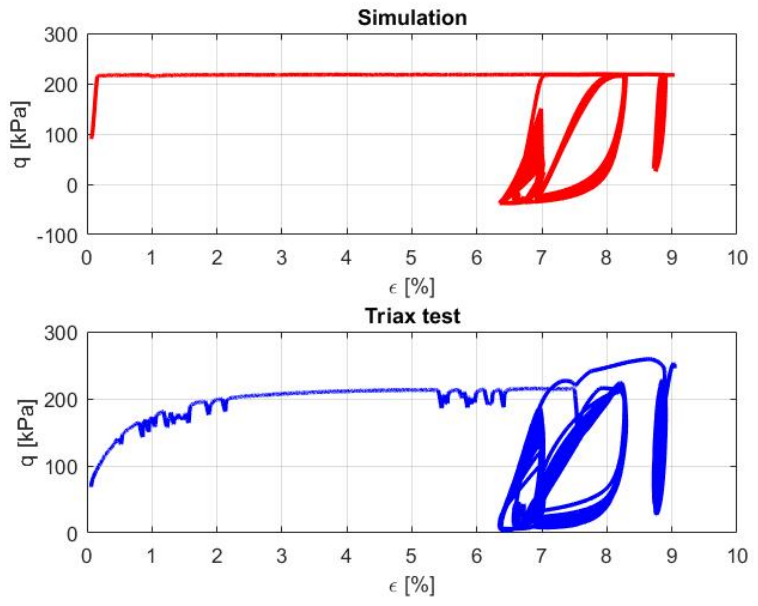

Fig. 8. Simulation and triaxial test where three different deformation amplitudes are applied (allowing 3 stiffness triangles to stabilize). The first triangle is $1.5 \%$ amplitude, $2^{\text {nd }}$ deformation cycle is $0.5 \%$ amplitude, and the $3^{r d}$ (last) is $0.25 \%$.

sity (volumetric response), which is ignored by BSM in its current implementation.

In Fig. 8 the deformation envelope is challenged to converge towards three different amplitudes: $1.5 \%$ amplitude at $7.5 \%$ mean, $1 \%$ amplitude at $6.5 \%$ mean, then $0.25 \%$ amplitude around $8.875 \%$ mean value. Thus, the $\epsilon_{y}$ envelope is challenged three times - the radius of the envelope is forced to increase (disturbed by yielding), then allowed to shrink (stabilize, converge). In the outcome of simulation, all three positions of stiffness triangles are visible. Note, the last triangle looks almost like a line (elastic). Alas, the illusion caused by unloading curve being steep, as the hysteresis loop is squeezed into a narrow $\epsilon_{y}$ envelope.

Tests in Fig's. 6-8 show sand response in drained mode. This means the ESP line is held in a constant posi- 

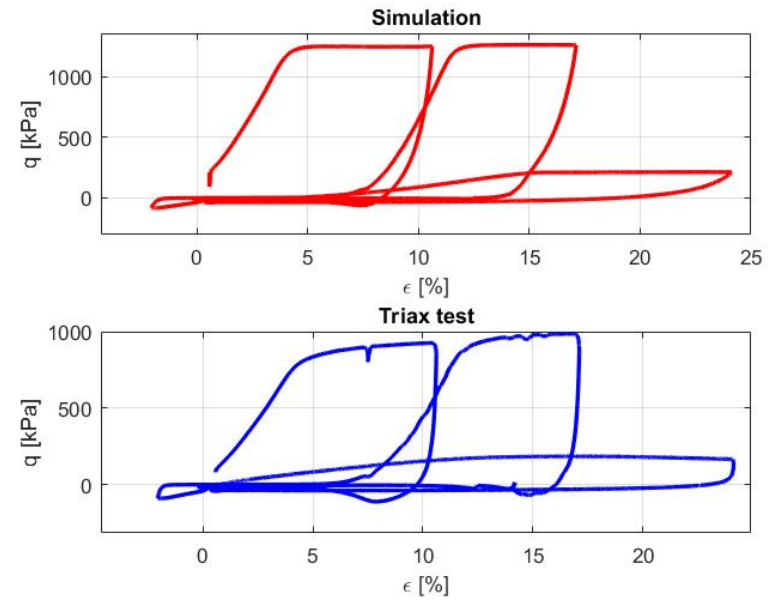

Fig. 9. Simulation and triaxial test where 3 loading cycles are applied at 3 different amplitudes. The first 2 cycles are applied undrained, the $3^{r d}$ - drained.

tion (see Fig.2), as $\sigma_{r}$ is constant. Test in Fig.9 has 2 cycles applied in undrained mode before the 3rd, drained, loading cycle. The overall shape and magnitude of response is predicted remarkably well by the simulation. Each cycle extend beyond the previous $\epsilon_{y}$ envelope limit, thus forcing the envelope to expand (the specimen gets disturbed towards a larger $\epsilon$ amplitude).

During undrained loading phases, $\sigma_{r}$ varies in Fig.9, due to variation in pore pressure, caused by the specimen contracting and dilating. Since volumetric response is not modeled, effective $\sigma_{r}$ vales are borrowed from measurement. Thus ESP line is moved along the $p^{\prime}$ axis using measured $\sigma_{r}$ values (see Fig.2). Thus, in the polar coordinates, only $\eta$ is a function of $\epsilon$, while $\sigma_{r}$ is a controlled by confining pressure (effective stress) independent of $\epsilon$ history. Alas, it is plausible that further research could link $\epsilon$ history with volumetric response [7], which would then allow to model the $\sigma_{r}$ component as a function of $\epsilon$ as well.

The final simulation example (Fig.10) contains an extremely complex array of irregular deformation cycles, combining both drained and undrained loading stages. The very plausibility of executing a triaxial test of such complexity is a recent state of the art accomplishment [6] [4] [5]. Now the BSM model, with the $\epsilon_{y}$ envelope is challenged to keep up with stress history developing during irregular loading cycles. As shown in Fig.10, the undrained peak are captured well, and the stiffness triangles continue to converge during drained phases of loading. At the very end of the test, in the far right of the plot, a drained stiffness triangle converges in both simulation and the triaxial test. Thus, the $\epsilon_{y}$ envelope has the remarkable capacity to re-calibrate itself, by converging towards a similar stiffness hysteresis loop as the specimen does.

\section{Conclusion}

The BSM approach is significantly different from convention. It explores a radically different approach, which is the
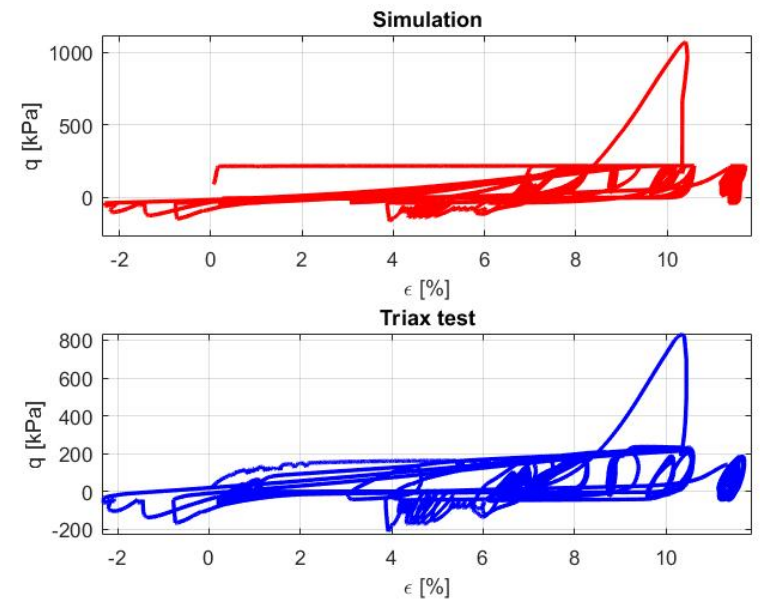

Fig. 10. Extremely complex loading sequence, tested using the frictionless triaxial apparatus. It contains both drained and undrained loading phases, liquefaction and post-liquefaction, during irregular loading cycles [6] [5].

exact opposite of convention: strain envelopes instead of stress envelopes, stress relaxation instead of plastic strains. Remarkably, the new approach allows to describe, predict and prescribe disturbed sand stiffness, in cyclic disturbed sand specimens.

A model using deformation $\left(\epsilon_{y}\right)$ rather than stress envelope is a novelty in geotechnics. A similar principle of strain-space plasticity has been attempted once before [8]. Alas, the authors of BSM were not aware of it. BSM was developed based exclusively on experiment observations [5] [6]. Up to this point, deformation dependent stiffness properties in disturbed sand were not explored in geotechnical paradigm. The common consensus is to model sand stiffness as a function of stress, rather than strain. Only volumetric response of frictional soil is widely considered to be a strain controlled problem [9] [7].

It is important to recognize that the physical phenomenon, which BSM is built on, is not predicted to exist by convention. The deformation dependent properties of converging stiffness hysteresis loops, are nether described nor predicted by convention. Nevertheless, BSM does not contradict well established conclusions within the existing geotechnical paradigm. BSM merely interpolates within known effective stress limits (Coulomb envelope) using a novel method - polar coordinates and a strain envelope. Remarkably, this allows to interpolate both drained and undrained stress history, during irregular loading cycles, using one set of rules.

\section{References}

[1] M. Damgaard, L. Andersen, L. Ibsen, H. Toft, J. Sørensen, Probabilistic Engineering Mechanics 41, 46-59 (2015)

[2] C.G. Di Prisco, D.M. Wood, Mechanical Behaviour of Soils Under Environmentallly-Induced Cyclic Loads, Vol. 534 (Springer Science \& Business Media, 2012) 
[3] R.E. Olson, J. Lai, Tech. rep., Chaoyang University of Technology (2004)

[4] T. Sabaliauskas, L. Ibsen, Cyclic Triaxial Loading of Cohesionless Silty Sand (International Society of Offshore and Polar Engineers, 2015), pp. 821-826, ISBN 978-1-880653-89-0

[5] T. Sabaliauskas, L.B. Ibsen et al., Triaxial testing beyond yielding, in The 27th International Ocean and Polar Engineering Conference (International Society of Offshore and Polar Engineers, 2017)

[6] S. Tomas, I. Lars, Bo, Geotechnical testing journal 42 (2019)

[7] R. Dobry, T. Abdoun, Journal of Geotechnical and Geoenvironmental Engineering p. 04015047 (2015)

[8] P.J. Yoder (1980), a Report on Research Concluded Under Grants from National Science Foundation

[9] R. Dobry, T. Abdoun, An investigation into why liquefaction charts work: A necessary step toward integrating the states of art and practice, in Proc., 5th Int. Conf. on Earthquake Geotechnical Engineering (Chilean Geotechnical Society Santiago, Chile, 2011), pp. $13-44$ 